\title{
All Together Now: Collective Intelligence for Computer-Supported Collective Action
}

\author{
Giuseppe Valetto*, Antonio Bucchiarone*, Kurt Geihs ${ }^{\dagger}$ Monika Buscher ${ }^{\ddagger}$, Katrina Petersen ${ }^{\ddagger}$, \\ Andrej Nowak ${ }^{\S}$, Agnieszka Rychwalska ${ }^{\S}$, Jeremy Pitt ${ }^{\Uparrow}$, Joseph Shalhoub \\ Francesca Rossill, Paolo Silingardi** and Paola Bernardeschi** \\ *Fondazione Bruno Kessler, Italy (valetto@fbk.eu, bucchiarone@fbk.eu) \\ łUniversity of Kassel, Germany (geihs@uni-kassel.de) \\ $\ddagger$ Lancaster University, UK (m.buscher@lancaster.ac.uk, k.petersen@lancaster.ac.uk) \\ §Warsaw University, Poland (andrzejn232@gmail.com, izziaczek@yahoo.com) \\ IImperial College London, UK (j.pitt@imperial.ac.uk, j.shalhoub@imperial.ac.uk) \\ "University of Pisa, Italy (frossi@math.unipd.it]) \\ **ACHAB Group, Italy (paolo.silingardi@achabgroup.it, paola.bernardeschi@achabgroup.it)
}

\begin{abstract}
In this position paper, we argue that an entirely new methodological paradigm and software platform is required for developing citizen-oriented social computing applications. The platform that we propose is based on the idea of 'Collective Intelligence as a Service', and is grounded in the formalisation of computational models derived from an empirical analysis of psychological processes and social practices. This in turn provides the enablers for developing radically innovative tools for computational sustainability and computer-supported collective action for Smart(er) Cities. The proposal is illustrated with two exemplars, one a healthcare application for patients with peripheral arterial disease; and the other an application for collaborative energy conservation to meet targets set out in a city's Sustainable Energy Action Plan.
\end{abstract}

\section{INTRODUCTION}

It was asserted by Mancur Olson, according to what came to be known as the zero contribution thesis, that "unless the number of individuals in a group is quite small, or unless there is coercion or some other special device to make individuals act in their common interest, rational, self-interested individuals will not act to achieve their common or group interests" [1, p. 2]. He therefore claimed that the argument that any 'large' group will ever organise itself to optimise a common goo is completely specious. This theoretical analysis was supported by the subsequent work of Hardin [2] on the 'tragedy of commons', essentially an $n$-player prisoners' dilemma which purports to show that a large group of actors will act to maximise their interests in the short term, even if that means depleting a common-pool resource which is in no-one's interest in the long term.

And yet, all the empirical evidence from quotidian experience and academic fieldwork [3], [4] demonstrates that "individuals in all walks of life and all parts of the world voluntarily organize themselves so as to gain the benefits of trade, to provide mutual protection against risk, and to create and enforce rules that protect natural resources" [5, p. 138]. The reality is that co-operative behaviour between (however empirically implausible) rational, self-interested individuals does occur, in large groups, and is sustainable over time although it is far from inevitable. It is also the case that top-down or external policy-making can frustrate, rather than reinforce such co-operation, if it interferes with the rights to self-organise [3].

In this position paper, we argue that in order to develop citizen-oriented social computing applications (cf. [6], [7]), while taking into account the size of group encountered in Smart Cities, leveraging developments in technology and communications, and accommodating external policy-making in a positive way, an entirely new methodological paradigm and software platform is required. The new methodological paradigm we propose is to ground the design of socio-technical systems in theories of group psychology and social practices. The new platform that we propose is based on the idea of 'Collective Intelligence as a Service', and is founded on formalisation of computational models derived from this empirical analysis of psychological processes and social practices. This in turn provides the enablers for developing radically innovative tools for computational sustainability and computersupported collective action for Smart(er) Cities.

Therefore, this paper is organised as follows. Section II discusses the background to this proposal in more detail, in particular with respect to the emerging (so-called) sharing economy. Section III presents the psychological and sociological bases for the methodological paradigm, while Section IV presents the technological basis for software platform. The proposal is illustrated with two exemplars in Section V, one a healthcare application for patients with peripheral arterial disease; and the other an application for collaborative energy conservation to meet targets set out in a city's Sustainable Energy Action Plan. We conclude in Section VI with some remarks about social computing with a social conscience.

\section{BACKGROUND: THE SHARING ECONOMY}

As the impact of disruptive ICT technologies keeps pervading our cities and our daily life, new opportunities (and risks) continuously arise, for individuals and communities alike. Many stem from a multitude of creative ways to leverage technical innovations as drivers and enablers to generate new social and economic models and mechanisms.

A paradigmatic example is the so-called sharing economy, which seems to have now reached an inflection point, and 
is beginning to significantly transform many socio-economic relationships and habits across environments, like Smart Cities, which provide a large-scale socio-technical milieu that is rich in opportunities and demand both technology- and participation-wise. The sharing economy is postulated on mobilizing and organizing a critical mass of citizens-actors, who - by operating in a peer-to-peer fashion - become at the same time providers and consumers (prosumers) of collectively provisioned services (either entirely novel, or, more often, alternative to analogous 'traditional' services provisioned according to a classic customer/supplier model). Sharing economy initiatives and systems are very visible examples of such Computer-Supported Collective Action [8] (CA) fostered by various forms of Collective Intelligence (CI) [9].

Instances of Computer-Supported CA applications and services abound in the sharing economy realm, and new ones seem to appear every day. However, many of these services present their participant and user communities with ethic and economic contradictions and dilemmas. Malhotra and Van Alstyne [10] offers a synthetic but exemplary catalog of such issues and problems, and how deeply they are embedded in how some of the fastest-rising and most popular sharing economy stars are organized and function.

We maintain that these problems inherently arise whenever the collective action instigated and supported by a given initiative is not sustainable. We define sustainable collective action initiative and services as those that:

- $\quad$ remain mindful of public policies of the social milieu they are operating in, as well as of priorities and shared concepts of collective welfare elaborated and agreed upon by the participant community; and,

- $\quad$ ensure that any generated benefits are distributed fairly by and between the participants themselves, that is, value diffuses to the edges of the peer-to-peer network, instead of accumulating disproportionately at some vantage positions in that network.

Only by maintaining these two properties can a CA application remain sustainable over time, that is, can continue to converge towards the priorities and goals established by its community, and avoid the emergence of disruptive dynamics that work against the community's goals. In contrast, a non-sustainable CA application will evolve in ways that may originate detrimental phenomena and pernicious network effects for the community.

We are therefore interested in fostering this property of sustainability in Computer-Supported CA. However, sustainability is a complex socio-technical characteristic, which can be only described and reasoned about using advanced concepts and instruments from the social sciences. It cannot - at this point - be translated in a technical property of an ICT solution (or a set thereof), and cannot be expressed in engineering design terms. To bridge that gap, we maintain that $a$ theoretical and methodological breakthrough is necessary. We need a systematic way to translate into formal computational models the theories and models of psychological and social processes that are conducive to sustainability in CA.

Moreover, that breakthrough should be embodied in a Collective Intelligence Engine(or CIE), a Collective Intelli- gence computing and service substrate that incorporates those formal computational models derived from sociology and psychology as native mechanisms that effectively facilitate building and operating CA applications, while ensuring their sustainable character.

We also envision that - in order to maximize its generative power and encapsulate the complexity of the underlying methodological innovation - the CIE should be delivered according to the architectural principles of Software-as-aService (SaaS), extend and specialized to achieve a design for Collective Intelligence as a Service (CIaaS).

\section{Social Perspectives}

Having theory that combines insights from group psychology and research into social practices would greatly advance such scholarship and practice. These two branches of social science will combine to produce novel, empirically grounded, and integrative theory with which to consider the problems of designing socio-technical CI systems.

\section{A. Dynamic Social Psychology}

To facilitate and foster collective action and minimize marginalization in Smart Cities, we need to understand how psychological and social processes combine to result in selforganization of social systems. Dynamical Social Psychology (DSP) [11] defines social groups as complex systems, where the interaction between heterogeneous individuals or subgroups results in self-organization and emergent properties at the system level. DSP incorporates empirically verified social psychological and cognitive mechanisms to study how different psychological and social variables acting as control parameters impact the macro, group-level properties [12], [13]. Applying the DSP approach to understand CA for Smarter Cities allows us to integrate both the findings of psychology: micro-level, individual determinants that have specific roles and effects in social practices but also system analysis that can point to policies that would optimize collective action and make it sustainable.

Computer modelling of social systems in the DSP approach may help tailor the developed Smart City systems in such a way that $\mathrm{CA}$ leads to collective intelligence and not to undesirable group level processes. In theory, the same set of individuals, with similar distribution of psychological traits can either form a constructive, task-oriented group or a singleminded mob. What could differentiate these two scenarios are interaction patterns and mechanisms governing the processes of social impact. Investigation of such scenarios might be close to impossible in controlled experiments, but a well-built agent based (based on psychological findings and theories rather than on direct extrapolation of physical models) could test various group interaction mechanism and provide invaluable insight into the design of CA platforms.

The Dynamical Social Impact Theory developed within the DSP paradigm shows how sustainable change may be introduced into social tissue while the minority and majority opinions survive and co-exist in a social system [14]. The generic, universal rules of agent (individual) interaction implemented in this model can be generalized into CA platforms. This will provide foundations to incorporate into ICT social psychology 
informed services for initiating and managing technologyinduced social change. Together, these approaches might show which interaction mechanisms and social influence processes help develop a Collective Intelligence system that properly incentivise engagement and participation of citizenry towards the achievement of given sustainable, community-defined CA policies and goals, while avoiding known detrimental phenomena, such as groupthink or crowd mentality, psychological biases or other pernicious network effects. It could establish which system designs promote productive diversification without collapse of social cohesion and alignment of action toward common goals without the simplifying unification of opinions and attitudes.

\section{B. Social Practices}

Research in sociology aims at understanding how the diverse groups of social actors who are stakeholders in a CA initiative can be engaged effectively in participatory policy synthesis. To begin with, this research shows that Collective Action is socially organised [15] and Collective Intelligence, too, is self-organising, synergetic collective reasoning and collaborative practice [9], which finds a necessary catalyst in digital technologies. CI, as a model of knowledge production, moves beyond the concept of knowledge bound to professional expertise, and instead situates intelligence in time, space, and practice. For example, such CI can be found in crowdsourcing and micro-tasking in platforms like Amazons Mechanical Turk [15] or Wikipedia [16], smartmobs who use digital technologies to coordinate protests or campaigns [17], and concepts that focus on peer production, open source software, and new digital economy 'commons' [18]. CI is a way in which diverse participants exert their voice and participate in science and technology decision- and policy-making[19]. Most importantly, these practices have the ability to produce societally transformative momentum, necessary for sustainable CA.

Unless the practices of collaboration and self-organizing of CI are promoted, tools intended to create and sustain CA will not be useful or sustainable in practice. To foster broad-based and effective CI and CA, we need to understand the underlying social practices and protocols for participation, reasoning, trust, recognition, and self-governance, and how to develop novel ICT that can make the most of this new relationship between expertise, media, public services and policy [20]. A particularly important question is how to integrate CIC tools that facilitate practices and processes for the self-organized setting, adjustment and evolution of agreed-upon community policies, which are a precursor and a foundation for any successful and sustainable CA.

System design needs to treat social practices in a way that goes beyond the 'ABC' of policy [21]. This is a reductionist view of policy that assumes that it is possible to simply collect and analyse data about attitudes (A) and behaviour (B) to influence choice (C). Considering the ways in which socio-technical systems combine the self-organized actions of many individuals to produce positive policy outcomes and on-going engagement in demographically, geographically and temporally shifting communities can help move beyond such inadequate models towards sustainable methods of participation and system design.
For the sociology perspective, then, CA is sustainable if system design: i) remains mindful of policies and priorities set by and for the participants in a way that focuses on motivation, trust, self-organisation, and sustainability; and, ii) ensures that any generated benefits are distributed fairly by and between the participants, via, for instance, participatory policy synthesis. Only with these two properties can a CA application remain sustainable over time, i.e., keep converging towards the policies and priorities established by its community, and avoid disruptive divergences that work against the community goals. Policy is a "science of muddling through" [22] that can be much improved, by leveraging CI to enable more robust and broad-based evidence and situated and participatory reasoning. To do so, we need investigations into how to facilitate the incorporation of social change instigated by collective intelligence into ethically aware collaboratively designed policy and goals [23].

\section{A Collective Intelligence Engine}

In order to develop citizen-oriented social computing applications for the Sharing Economy, we argue that an innovative software platform is required, like the Collective Intelligence Engine depicted in Figure 1. Such a platform would support the concept (and demonstrate the value) of Collective Intelligence as a Service (CIaas) in Collective Intelligence Computing, and act as a generic technology that supports sustainable collective action.

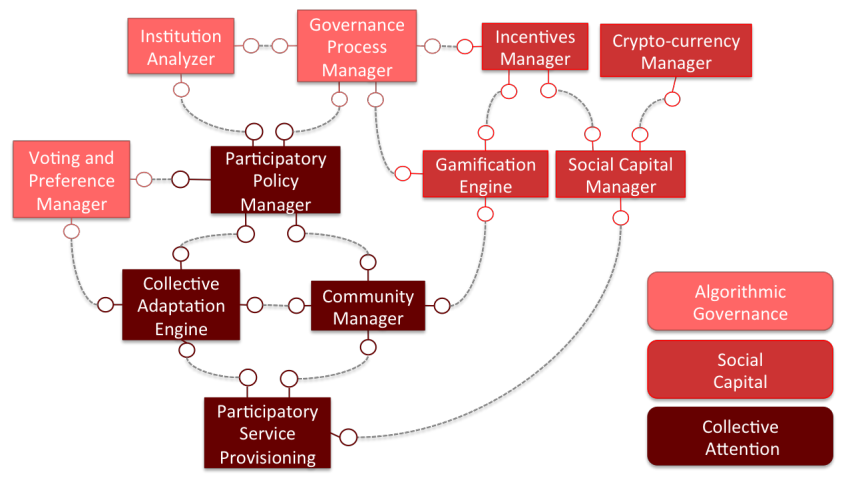

Fig. 1: Collective Intelligence Engine

The development of the CIE derives from three complementary aspects: Social Capital, Algorithmic Governance, and Collective Attention.

Social Capital is a form of CIC that aims at representing and working with social capital in self-organising sociotechnical systems. Social capital is an attribute of individuals that enhances their ability to solve collective action problems, which may take different forms, for example reputation, social networking and institutions [24]. Each of these forms is a subjective indicator of one individual's expectations of how other individuals will behave in a $n$-player cooperative dilemma. In the pursuit of successful and sustainable CA, understanding the social practices that lead to the creation of social capital, the group psychology that gives a valuation of social capital, and the mechanisms by which social capital achieves community goals [5], [25].

For this pursuit, we argue that any CIE needs to be based in a recent framework for electronic social capital [26] that 
defines data structures to represent mechanisms using those metrics as input, needs to draw upon a gamification approach that captures, in algorithmic form, suistainable policies and practices, and an implementation of electronic social capital as crypto-currency in a way that operates on the exchange of social capital as incentive.

In detail, it includes a Social Capital Manager that acts as a decision-support system for actors in the collective action community; an Incentive Manager that sets, reason about and modifies the system of incentives within the community; a Gamification Engine that uses policies, incentives and rules of governance (provided by the Algorithmic Governance) to promote at the Collective Attention a range of sustainable community behaviours; and a Crypto-currency Enables that manages any intra-community crypto-currency conversions and transactions.

Algorithmic governance is a form of CIC that seeks to represent, and reason with, rules and processes of deliberation and decision-making in computational form. In socio-technical systems, it is necessary to ensure that those affected by such rules and processes participate in their selection and modification. The objective is to formalize good governance that encourages (socially) good outcomes.

To this ends, in our CIE, we incorporate algorithmic governance socio-psychological principles, politico-economic principles by translating into computational form the social practices and psychological mechanisms that influence the governance of the various aspects of collective action, in ways that enhance the prospect of its success and sustainability. Specifically, the algorithmic governance outcomes are applied to the processes and functions of community that are managed by the Collective Attention.

Algorithmic governance, as depicted in Figure 1, is realized thanks to different enablers devoted to develop a comprehensive computational model of self-organized collective governance. In particular, using computational logic, starting from the formal characterisation of Ostrom's institutional design principles [3], and extending that with principles and processes of group psychology and social practices. The main objective here is to investigate how institutions form, self-organise their functions, structures, and components over time, and sustain themselves over with respect to achieving the legitimate purposes for which they were founded. This is achieved thanks to three main enablers: a Governance Process Manager which offers a library of protocols for configuring typical governance aspects - such as access control, deliberative assembly and dispute resolution - of organizational and CA practices; an Institution Analyser which supports run-time evaluation of institutions and their forms, according to criteria such as transparency, fairness/justice and openness; and a Voting and Preference Manager which offers secure non-manipulatable, non-repudiatable electronic voting and preference determination facility.

Collective Attention is a form of Collective Intelligence Computing (CIC) that addresses the process of how a community having a joint interest in some high-level goal can reflect on the current state of achievement of its goals, and how it can can reason about how their actions may contribute to achieving their community-wide goal. Collective attention transforms individual observations into a shared greater whole, which provides feedback to the participants on the state of their collective affairs.

Research challenges in Collective Attention are to provide models and techniques of how collective attention, action, and adaptation are performed and interconnected. This includes micro-level modeling pertaining to the individual actors, and macro level modelling of how group practices emerge from composition of activities at the individual level. The representation of composition operators and how to apply them represents a major open research challenge for this aspect. In more details, mechanisms to handle how different individuals become part of the same and emergent community, how they collectively devise and provision services to the community, and how they collectively adapt to changes in policies (i.e., Collective Adaptation [27]), context, incentives and opportunities, are needed. This is done through a Community Manager to handle emergent groups and individual roles, a Participatory Service Provisioning Module that facilitates the creation of new collective services, and a Collective Adaptation Engine that supports adaptation to changes in a community.

All the techniques described above are informed and complemented by models and mechanisms of Algorithmic Governance and by models and mechanisms around incentives and and social capital from Social Capital.

\section{TWO EXEMPLARS}

In this section, we illustrate the ideas of CIaaS with two exemplar applications, the first in healthcare, and the second in sustainable energy.

\section{A. Healthcare Application}

One exemplar for CIaaS is a group self-assistance application within a healthcare setting, by coordinating individuals with peripheral arterial disease in self-organised (self)supervised exercise, with the collective goal of improving lower limb symptoms, quality of life, distance walked, overall cardiovascular health, and reducing the need for and costs of invasive treatments, insurance premiums and payouts.

Supervised exercise is a recommended treatment for patients with intermittent claudication (pain in the calf/calves with exertion owing to arterial disease of the lower limbs). The benefit of medically supervised exercise programmes, in terms of symptoms, quality of life and distance walked are supported by a strong (level 1) evidence base. For example, it has been found that a weekly, supervised exercise and motivation class for a 6-month period provides a significant improvement in patients' symptoms, quality of life, and distance walked compared with advice alone and this improvement continues after attendance at class has ceased [28]. In fact, the use of supervised exercise for individuals with peripheral arterial disease manifesting as intermittent claudication is in line with current evidence, and international best practice and (grade A) consensus guidelines (see e.g. [29]).

However, vascular surgeons correctly recognise that many patients presenting with intermittent claudication and peripheral arterial disease are best treated by supervised exercise rather than endovascular or surgical intervention; but that doing 
the exercise causes short-term pain, and patients experiencing pain tend to stop doing whatever causes the pain, unless they are supervised. Such supervision is, though, impractical, given the scale of the problem: peripheral arterial disease affects 7$14 \%$ of the UK population, is responsible for $2 \%$ of hospital admissions, and furthermore, $2 \%$ of individuals with diabetes develop a foot ulcer annually.

Therefore, addressing peripheral arterial disease is a significant and unmet clinical and health economic need, but the treatment of intermittent claudication using supervised exercise remains largely under-utilised due to a lack of appropriate resources. Therefore, we propose an alternative approach based on self-organised, self-supervised exercise using CIaaS.

To develop such an application requires identification of the principles of social practices and of dynamic social psychology that are involved in the establishment and management of selforganising 'self-help communities'. For example, the Bubble Theory of Social Change [30] specifies how a sustainable social change may be achieved by concentrating on changing fragments of social networks (clusters or bubbles) rather than separate individuals. Our belief is that the successful transition from supervised exercise to self-supervised exercise is a process of social change rather than simply a transfer of knowledge, responsibility or roles, and that this process can be organised (and self-organised) as in, for example, the community running events organised by parkrun (www.parkrun.org.uk).

The next step in developing the application requires understanding the roles of these self-help communities in the wider context. It has been observed that complex social systems are not necessarily chaotic, but are organised in some productive fashion, with multiple stakeholders and polycentric governance (multiple centres of decision-making) [31]. This will entail identifying all the relevant stakeholders (e.g. clinicians, health service, policy advisors, and insurance providers) and understanding their co-dependence (cf. [32] for community energy systems), and instantiating governance services for a peerto-peer sharing economy with distributed decision-making between the stakeholders.

The final step will entail defining services for provision and appropriation of social capital with respect to social practices, and for collective awareness with respect to wellknown psychological biases about attention (e.g. what have been referred to as the single action bias (where an individual performs one participatory action and deems it sufficient) and the the omission bias (where an individual feels worse about an inappropriate action than inaction) [33]).

If successful, we would expect this application would demonstrate that the health benefits of self-supervised exercise approximate those of supervised programmes, but with vastly improved availability and cost effectiveness.

\section{B. Sustainable Energy Action Plans}

An application domain which has high-impact potential and can lead to long-term, sustainable benefits in a Smart City is that collaborative energy conservation. TA case study in this domain can involve a variety of stakeholders and actors in the city, ranging from the city administration, to service providers and commercial and industrial players in and around the energy value chain, local associations and grassroots groups, all the way to households and individual citizens.

A case study in this domain can be framed in the context of Smart Citys Sustainable Energy Action Plan (SEAP), as per the Covenant of Mayors initiative within the EU 20-20-20" effort, to which a large number of cities in Europe are subscribing. The main objective of the CIE in this areas would be to enable the development of citywide campaigns and fostering the growth of grassroots initiatives that can effectively mobilize the energies and creativity of citizens towards achieving the conservation targets set forth in a SEAP.

Achieving SEAP goals cannot be done only by top-down planning and policy implementation by the city administration alone. They are typically very ambitious and inherently require the active engagement of citizens, individually and collectively, in embracing best practices of energy conservation put forth by the city, as well as devising new such practices. Therefore this case study must have the goal to encourage citizens to come together, in order to adhere to, design, implement and improve energy conservation initiatives, with a special focus on those initiatives that benefit from a network effect, or that become effective only when agreed upon by groups of different size (e.g. apartment buildings, neighborhoods, businesses, etc.).

A main focus in such a Computer-Supported CA application is to foster the ability and opportunity of citizens to selforganize in the form of collectively provided services ( $a$ la sharing economy), through which citizens can offer expertise and facilitate the exchange, diffusion and adoption of virtuous energy conservation practices by other fellow citizens. Another important focus is the exploration of games and gamification, as a way to incentivize and boost virtuous behaviors in a collaborative/competitive fashion, through which participants can gain status, reputation and rewards.

The combination of collectively provided services and gamified incentives models can be further augmented by the use of crypto-currencies as a fungible reification of incentives and rewards, which can be transacted among the various actors, and even with the city administration in lieu of or as a coupon towards service fees or monies.

\section{SUMMARY AND CONCLUSIONS}

Computer-supported collective action, and more in general social computing, is the next upcoming wave of ICT-induced innovation that will permeate society. Phenomena like the sharing economy, crowd-sourcing, crowd-funding and many others are only early signs of how social computing has a potential of stronger and more deeply embedded effects in society than previous waves. This is, because, though sociotechnical interactions, it causes the fabric of society itself - starting from with inter-personal relationships to crosscommunity interactions - to become embodied in technological design, i.e., to become digitized.

The proposal presented in this paper is in a position to fundamentally impact this, because of its inter-disciplinary approach, the identification of the concept of sustainability as a key property for success of computer-supported CA, and the unique combination of algorithmics for governance, collective attention and social capital. This can effectively translate a 
deeper understanding of social practices and psychological dynamics from the sociology and psychology of sustainable CA into computational form, and which are well-positioned to be developed into a CIC framework.

A wide variety of stakeholders are and will be interested in being able to build computer-supported CA systems and applications, ranging from public administrations, to businesses, to emergent communities, to tech-savvy philanthropists and activists etc. However, nobody knows yet how to build systems of this kind effectively, although we know that CIC represent a central cog of their design. In particular, to date, principles and patterns to architect CA initiatives that respect and promote the common good have not been fully articulated or established. We argue that something like ClaaS is essential if we are to develop computer-supported CA applications that take ethics, welfare, group dynamics, benign governance and (best) social practices into account: what we call social computing with a social conscience.

\section{REFERENCES}

[1] M. Olson, The Logic of Collective Action. Harvard University Press, 1965.

[2] G. Hardin, "The tragedy of the commons," Science, vol. 162, no. 3859, pp. 1243-1248, Dec. 1968.

[3] E. Ostrom, Governing the Commons. Cambridge University Press, 1990.

[4] D. Bollier and S. Helfrich, Eds., The Wealth of the Commons: A World Beyond Market and State. Levellers Press, 2012.

[5] E. Ostrom, "Collective action and the evolution of social norms," The Journal of Economic Perspectives, vol. 14, no. 3, pp. 137-158, 2000.

[6] F. Giunchiglia and D. Robertson, "The social computer: Combining machine and human computation," University of Trento, Tech. Rep. DISI-10-036, 2010

[7] D. Miorandi, V. Maltese, M. Rovatsos, A. Nijholt, and J. Stewart, Eds., Social Collective Intelligence: Combining the Powers of Humans and Machines to Build a Smarter Society. Springer, 2014.

[8] A. Shaw, H. Zhang, A. Monroy-Hernández, S. Munson, B.M, Hill, E. Gerber, P. Kinnaird, and P. Minder, "Computer supported collective action," ACM Interactions, vol. 21, no. 2, pp. 74-77, March 2014.

[9] P. Levy, Collective Intelligence. Mankind's Emerging World in Cyberspace. Cambridge, MA. USA: Perseus Books, 1997.

[10] A. Malhotra and M. Van Alstyne, "The dark side of the sharing economy... and how to lighten it," Communications of the ACM, vol. 57, no. 11, pp. 24-27, 2014.

[11] A. Nowak and R. Vallacher, Dynamical social psychology. Guilford Press, 1998.

[12] B. Latané, A. Nowak, and J. Liu, "Measuring emergent social phenomena: Dynamism, polarization, and clustering as order parameters of social systems," Behavioral science, vol. 39, no. 1, pp. 1-24, 1994.

[13] R. Vallacher, S. Read, and A. Nowak, "The dynamical perspective in personality and social psychology," Personality and Social Psychology Review, vol. 6, no. 4, pp. 264-273, 2002.

[14] A. Nowak, J. Szamrej, and B. Latane, "From private attitude to public opinion: a dynamic theory of social impact," Psychological Review, vol. 97, pp. 362-376, 1990.
[15] Y. Benkler, A. Shaw, and B. Hill, "Peer production: A modality of collective intelligence," in The Collective Intelligence Handbook, T. Malone and M. Berntstein, Eds. MIT Press, to appear.

[16] C. Leadbeater, We-think. Mass Innovation, not Mass Production. Profile Books, 2008.

[17] H. Rheingold, Smart Mobs: The next social revolution. Perseus Books, 2003.

[18] S. Macbeth and J. Pitt, "Self-organising management of user-generated data and knowledge," The Knowledge Engineering Review, vol. FirstView, no. 1-28, 2014.

[19] M. Michael, "Publics performing publics: of pigs, pips and politics," Public Understanding of Sceince, vol. 18, no. 5, pp. 617-631, 2009.

[20] M. Buscher, M. Liegl, and V. Thomas, "Intelligence in crises," in Social collective intelligence: combining the powers of humans and machines, J. Stewart, Ed. Springer, 2014.

[21] E. Shove, "Beyond the abc: climate change policy and theories of social change," Environment and Planning A, vol. 42, no. 6, pp. 1273-1285, 2010.

[22] R. Scott, "The science of muddling through revisited," Emergence Complexity and Organization, vol. 1, no. 5-18, 12.

[23] M. Hartswood, B. Grinmpe, M. Jirotka, and S. Anderson, "Towards the ethical governance of smart society," in Social Collective Intelligence: Combining the Powers of Humans and Machines to Build a Smarter Society, D. Miorandi, V. Maltese, M. Rovatsos, A. Nijholt, and J. Stewart, Eds. Springer, 2014.

[24] E. Ostrom and T. K. Ahn, "Introduction," in Foundations of Social Capital, E. Ostrom and T. K. Ahn, Eds. Cheltenham, UK: Edward Elgar Publishing, 2003, pp. xi-xxxix.

[25] S. Bowles and H. Gintis, "Social capital and community governance," The Economic Journal, vol. 112, no. 483, pp. F419-F436, 2002.

[26] P. E. Petruzzi, D. Busquets, and J. V. Pitt, "Experiments with social capital in multi-agent systems," in PRIMA 2014: Principles and Practice of Multi-Agent Systems, 2014, pp. 18-33.

[27] A. Bucchiarone, C. Mezzina, M. Pistore, H. Raik, and G. Valetto, "Collective adaptation in process-based systems," in Eighth IEEE International Conference on Self-Adaptive and Self-Organizing Systems, SASO 2014, London, United Kingdom, September 8-12, 2014, 2014, pp. $151-156$.

[28] D. Cheetham, L. Burgess, M. Ellis, A. Williams, R. Greenhalgh, and A. Davies, "Does supervised exercise offer adjuvant benefit over exercise advice alone for the treatment of intermittent claudication? a randomised trial," European Journal of Vascular and Endovascular Surgery, vol. 27, pp. 17-23, 2004.

[29] M. Conte, F. Pomposelli, D. Clair, P. Geraghty, J. McKinsey, J. Mills, G. Moneta, H. Murad, R. Powell, A. Reed, A. Schanzer, and A. Sidawy, "Society for vascular surgery practice guidelines for atherosclerotic occlusive disease of the lower extremities: Management of asymptomatic disease and claudication," Journal of Vascular Surgery, vol. 61, no. 3, pp. 2S-41S, 2015 .

[30] A. Nowak, M. Lewenstein, and J. Szamrej, "Bable modelem przemian spolecznych (bubbles: a model of social transition)," Swiat Nauki (Scientific American Polish Edition), vol. 12, 1993.

[31] E. Ostrom, "Beyond markets and states: Polycentric governance of complex economic systems," in Les Prix Nobel. The Nobel Prizes 2009, K. Grandin, Ed. Stockholm: Nobel Foundation, 2010, pp. 408-444.

[32] A. Diaconescu and J. Pitt, "Holonic institutions for multi-scale polycentric self-governance," in Proceedings COIN. Springer, to appear.

[33] A. Nowak, A. Rychwalska, and J. Szamrej, "Social, psychological and technological determinants of energy use," IEEE Technology \& Society Magazine, vol. 33, no. 3, pp. 42-47, 2014. 